\title{
Uniqueness of Closed Equilibrium Hypersurfaces for Anisotropic Surface Energy and Application to a Capillary Problem
}

\author{
Miyuki Koiso \\ Institute of Mathematics for Industry, Kyushu University, Motooka Nishi-ku, Fukuoka 819-0395, Japan; \\ koiso@math.kyushu-u.ac.jp
}

Received: 22 September 2019; Accepted: 6 October 2019; Published: 10 October 2019

\begin{abstract}
We study a variational problem for hypersurfaces in the Euclidean space with an anisotropic surface energy. An anisotropic surface energy is the integral of an energy density that depends on the surface normal over the considered hypersurface, which was introduced to model the surface tension of a small crystal. The purpose of this paper is two-fold. First, we give uniqueness and nonuniqueness results for closed equilibria under weaker assumptions on the regularity of both considered hypersurfaces and the anisotropic surface energy density than previous works and apply the results to the anisotropic mean curvature flow. This part is an announcement of two forthcoming papers by the author. Second, we give a new uniqueness result for stable anisotropic capillary surfaces in a wedge in the three-dimensional Euclidean space.
\end{abstract}

Keywords: anisotropic mean curvature; anisotropic surface energy; Wulff shape; anisotropic mean curvature flow; crystalline variational problem; capillary problem

\section{Introduction}

We study equilibrium hypersurfaces in the $(n+1)$-dimensional Euclidean space $\mathbb{R}^{n+1}$ for anisotropic surface energy, which serve as a mathematical model of small crystals and small liquid crystals with anisotropy. Therefore, the objects of our study should be not only smooth hypersurfaces but also hypersurfaces with singular points like vertices and edges. However, such equilibrium hypersurfaces with both smooth curved parts and singular points have not yet been studied sufficiently well. One of the purposes of this paper is to give a new concept a piecewise- $C^{2}$ weak immersion and to study variational problems in this class of hypersurfaces. Piecewise- $C^{2}$ weak immersions are hypersurfaces with the weakest regularity in order to study this type of variational problem by using essentially classical differential geometry. The other purpose of this paper is to give a new uniqueness result for stable anisotropic capillary surfaces in a wedge in $\mathbb{R}^{3}$, which is proved by using the method developed to pursue the first purpose together with careful treatment of the boundary part by using several new formulas.

Let $\gamma: S^{n} \rightarrow \mathbb{R}_{>0}$ be a positive continuous function on the unit sphere $S^{n}=\left\{v \in \mathbb{R}^{n+1} ;\|v\|=1\right\}$ in $\mathbb{R}^{n+1}$. Let $X$ be a closed hypersurface in $\mathbb{R}^{n+1}$ for which the tangent hyperplane is well defined at almost every point. $X$ will be represented as a mapping $X: M \rightarrow \mathbb{R}^{n+1}$ from an $n$-dimensional oriented connected compact $C^{\infty}$ manifold $M$ into $\mathbb{R}^{n+1}$. Let $v$ be the unit normal vector field along $\left.X\right|_{M \backslash S[X]}$, where $S[X]$ is the set of singular points of $X$. The anisotropic energy $\mathcal{F}_{\gamma}(X)$ of $X$ is defined as $\mathcal{F}_{\gamma}(X):=\int_{M \backslash S[X]} \gamma(v) d A$, where $d A$ is the $n$-dimensional volume form of $M$ induced by $X$. Such an energy was introduced by Gibbs (1839-1903) in order to model the shape of small crystals, and it is used as a mathematical model of anisotropic surface energy [1,2]. It is known that, for any positive number $V>0$, among all closed hypersurfaces as above enclosing the same $(n+1)$-dimensional 
volume $V$, there exists a unique (up to translation in $\mathbb{R}^{n+1}$ ) minimizer $W_{\gamma}(V)$ of $\mathcal{F}_{\gamma}$ [3]. The minimizer $W_{\gamma}\left(V_{0}\right)$ for the specific value $V_{0}:=(n+1)^{-1} \int_{S^{n}} \gamma(v) d S^{n}$ is called the Wulff shape for $\gamma$, and we will denote it by $W_{\gamma}$. When $\gamma \equiv 1, \mathcal{F}_{\gamma}(X)$ is the usual $n$-dimensional volume of the hypersurface $X$ and $W_{\gamma}$ is the unit sphere $S^{n}$.

In this paper, we study variational problems for the anisotropic energy $\mathcal{F}_{\gamma}$. In general, the Wulff shape and equilibrium hypersurfaces of such an energy for volume-preserving variations are not smooth. In view of the origin of the energy functional mentioned above, it is natural to try to study the problem under the lowest possible regularities of the considered hypersurfaces and the energy density function. In order to pursue it, we define a new concept "piecewise- $C^{2}$ weakly immersed hypersurface" and study the variational problem of the energy $\mathcal{F}_{\gamma}$ for $C^{2}$ functions $\gamma$ on $S^{n}$. These assumptions on the regularity are weaker than any previous works that studied variational problems of anisotropic surface energies in differential geometry (cf. References [4-9]). Under such a weak regularity assumption, we concentrate upon the problem of uniqueness for closed equilibrium hypersurfaces. In order to give precise statements of our results, we prepare a few words.

For any given convex set $\tilde{W}$ having the origin of $\mathbb{R}^{n+1}$ inside, there exists a Lipschitz continuous function $\gamma: S^{n} \rightarrow \mathbb{R}_{>0}$ such that the boundary $W:=\partial \tilde{W}$ of $\tilde{W}$ coincides with the Wulff shape $W_{\gamma}$ for $\gamma$. However, such $\gamma$ is not unique. The "smallest" $\gamma$ is called the convex integrand for $W$ (or, simply, convex) (for another equivalent definition, see Section 2).

Each equilibrium hypersurface $X$ of $\mathcal{F}_{\gamma}$ for variations that preserve the enclosed $(n+1)$-dimensional volume (we will call such a variation a volume-preserving variation) has constant anisotropic mean curvature. Here, the anisotropic mean curvature $\Lambda$ of a piecewise- $C^{2}$ hypersurface $X$ is defined at each regular point of $X$ as (cf. Reference [6,10]) $\Lambda:=(1 / n)\left(-\operatorname{div}_{M} D \gamma+n H \gamma\right)$, where $D \gamma$ is the gradient of $\gamma$ and $H$ is the mean curvature of $X$. If $\gamma \equiv 1, \Lambda=H$ holds.

We call a piecewise- $C^{2}$ weakly immersed equilibrium hypersurface $X$ a CAMC (constant anisotropic mean curvature) hypersurface (see Definition 3 for details). A CAMC hypersurface is said to be stable if the second variation of the energy $\mathcal{F}_{\gamma}$ for any volume-preserving variation is nonnegative.

One of the most basic questions is as follows:

\section{Question 1. Is any closed CAMC hypersurface the Wulff shape?}

The answer to this uniqueness problem is not affirmative in general [11-13]. However, it is expected that, if one of the following "good" conditions (I)-(III) is satisfied, the image of any closed CAMC hypersurface $X$ coincides with the Wulff shape (up to translation and homothety).

(I) $X$ is an embedding; that is, $X$ is an injective mapping.

(II) $X$ is stable.

(III) $n=2$ and the genus of $M$ is 0 ; that is, $M$ is homeomorphic to $S^{2}$.

If we assume that $W_{\gamma}$ is a smooth, strictly convex hypersurface (that is, the support function $\gamma$ of $W_{\gamma}$ is strictly convex. See Definition 1.), any closed CAMC hypersurface $X$ is also smooth and the above expectation was already proved. In fact, if $X$ satisfies one of (I)-(III), it is a homothety of the Wulff shape (which was proved by the following papers: For (I), Reference [14] for $\gamma \equiv 1$ and Reference [5] for general $\gamma$; for (II), Reference [15] for $\gamma \equiv 1$ and Reference [8] for general $\gamma$; and for (III), Reference [16] for $\gamma \equiv 1$ and Reference [7] for general $\gamma$ ). However, the situation is not the same for more general $\gamma$ and/or $W_{\gamma}$. Actually, if $\gamma$ is not strictly convex, even if it is convex (See Definition 1) and of class $C^{\infty}$, the Wulff shape can have singular points and its principal curvatures can be unbounded (Example 3). Also, we have the following striking nonuniqueness results.

Theorem 1 ([17]). There exists a $C^{\infty}$ function $\gamma: S^{n} \rightarrow \mathbb{R}_{>0}$ such that there exist closed embedded CAMC hypersurfaces in $\mathbb{R}^{n+1}$ for $\gamma$, each of which is not (any homothety or translation of) the Wulff shape $W_{\gamma}$. 
Theorem 2 ([17]). There exists a $C^{\infty}$ function $\gamma: S^{2} \rightarrow \mathbb{R}_{>0}$ such that there exist closed embedded CAMC surfaces in $\mathbb{R}^{3}$ with genus zero for $\gamma$, each of which is not (any homothety or translation of) the Wulff shape $W_{\gamma}$.

Theorems 1 and 2 are proved by giving suitable examples (Section 4 ). The same examples give self-similar shrinking solutions with genus 0 for anisotropic mean curvature flow, which are not (any homotheties or translations of) the Wulff shape (Section 6). In contrast with this, the round sphere is the only closed embedded self-similar shrinking solution of the mean curvature flow in $\mathbb{R}^{3}$ with genus zero [18].

As for the uniqueness of stable closed CAMC hypersurfaces, we obtain the following result.

Theorem 3 ([19]). Assume that $\gamma: S^{n} \rightarrow \mathbb{R}_{>0}$ is of class $C^{2}$ and convex. Then, the image of any closed stable piecewise- $C^{2} C A M C$ hypersurface for $\gamma$ of which the rth anisotropic mean curvature for $\gamma($ see Section 3 ) is integrable for $r=1, \ldots, n$ is (up to translation and homothety) a covering of the Wulff shape $W_{\gamma}$.

Let us mention the preceding works relating to Theorem 3. As for planer curves, Morgan [20] proved that, if $\gamma: S^{1} \rightarrow \mathbb{R}_{>0}$ is continuous and convex, then any closed equilibrium rectifiable curve for $\mathcal{F}_{\gamma}$ in $\mathbb{R}^{2}$ is (up to translation and homothety) a covering of the Wulff shape. About uniqueness of closed stable equilibria in $\mathbb{R}^{3}$, Palmer [9] proved the same result as Theorem 3 but under the assumptions that $\gamma$ is of $C^{3}$ and that considered surfaces and the Wulff shape satisfy some extra assumptions.

A similar method to prove Theorem 3 together with careful treatment on the free boundary gives a uniqueness result for a capillary problem. For simplicity, we assume that $\gamma: S^{2} \rightarrow \mathbb{R}_{>0}$ is a strictly convex function of class $C^{\infty}$. Let $\Omega$ be a wedge-shaped domain bounded by two planes $\Pi_{1}, \Pi_{2}$ in $\mathbb{R}^{3}$, and let $\omega$ be a positive constant. Let $M$ be a two-dimensional oriented connected compact $C^{\infty}$ manifold with boundary $\partial M=\sigma_{1} \cup \sigma_{2}$, where each $\sigma_{j}$ is a topological circle. Consider any $C^{\infty}$-immersion $X:\left(M, \sigma_{1}, \sigma_{2}\right) \rightarrow\left(\bar{\Omega}, \Pi_{1}, \Pi_{2}\right)$ of which the restriction $\left.X\right|_{\partial M}$ to $\partial M$ is an embedding. Set $C_{j}=X\left(\sigma_{j}\right)$, and let $D_{j} \subset \Pi_{j}$ be the domain bounded by $C_{j}(j=1,2)$. We define the wetting energy $\mathcal{W}(X)$ of $X$ as follows:

$$
\mathcal{W}(X)=\omega\left(\mathcal{H}^{2}\left(D_{1}\right)+\mathcal{H}^{2}\left(D_{2}\right)\right),
$$

where $\mathcal{H}^{2}\left(D_{i}\right)$ is the area of $D_{i}$. Then, we define the total energy $E(X)$ of $X$ by

$$
E(X)=\mathcal{F}_{\gamma}(X)+\mathcal{W}(X)
$$

Note that $X(M) \cup D_{1} \cup D_{2}$ is a piecewise smooth surface without boundary. We denote by $V(X)$ the oriented volume enclosed by $X(M) \cup D_{1} \cup D_{2}$. We call a critical point of $E$ for volume-preserving variations an anisotropic capillary surface (or, simply, a capillary surface). A capillary surface is said to be stable if the second variation of $E$ is nonnegative for all volume-preserving variations of $X$. In the following uniqueness theorem, we identify $M$ with $X(M)$.

Theorem 4. Let $\Omega$ be a wedge in $\mathbb{R}^{3}$ bounded by two planes $\Pi_{1}$ and $\Pi_{2}$, and let $M \subset \bar{\Omega}$ be a compact oriented immersed surface that is disjoint from the edge $\Pi_{1} \cap \Pi_{2}$ of $\Omega$, having embedded boundary $\partial M \subset \Pi_{1} \cup \Pi_{2}$ and satisfying $\partial M \cap \Pi_{j}=\partial D_{j}$ for a nonempty bounded domain $D_{j}$ in $\Pi_{j}$. If $M$ is a stable anisotropic capillary surface in $\bar{\Omega}$ and both $D_{1}$ and $D_{2}$ are convex, then $M$ is (up to translation and homothety) part of the Wulff shape $W_{\gamma}$. Conversely, if $M$ is part of $W_{\gamma}$ (up to translation and homothety), then it is stable.

As for previous works which are closely related to Theorem 4, we have the following. We studied the existence and uniqueness of stable anisotropic capillary surfaces between two parallel planes $\Pi_{1}$ and $\Pi_{2}$ in $\mathbb{R}^{3}$ [21-23]. Moreover, in Reference [24], we proved the uniqueness result similar to Theorem 4 for isotropic capillary hypersurfaces in a wedge in $\mathbb{R}^{n+1}$; here, isotropic means that $\gamma \equiv 1$.

This article is organized as follows. In Section 2, we give the definition and a representation of the Wulff shape and some fundamental concepts relating to the Wulff shape. Also some typical examples 
are given. In Section 3, we give the Euler-Lagrange equations for our variational problem and the definitions of anisotropic curvatures. In Section 4, we give outlines of the proofs of Theorems 1 and 2. In Section 5, an outline of the proof of Theorem 3 is given. In Section 6, we mention an application to anisotropic mean curvature flow. In Section 7 , we give the proof of Theorem 4.

\section{Preliminaries}

Let $\gamma: S^{n} \rightarrow \mathbb{R}_{>0}$ be a positive continuous function. In this paper, we call the boundary $W_{\gamma}$ of the convex set $\tilde{W}[\gamma]:=\cap_{v \in S^{n}}\left\{X \in \mathbb{R}^{n+1} ;\langle X, v\rangle \leq \gamma(v)\right\}$ the Wulff shape for $\gamma$, where $\langle$,$\rangle means the$ standard inner product in $\mathbb{R}^{n+1}$. In other literatures, $\tilde{W}[\gamma]$ is often called the Wulff shape.

From now on, any parallel translation of the Wulff shape $W_{\gamma}$ will be also called the Wulff shape, and it will be denoted also by $W_{\gamma}$, if it does not cause any confusion.

Let us define two terminologies which represent the convexity of the energy density function $\gamma$.

Definition 1. (i) (cf. References [3,25]) A continuous map $\gamma: S^{n} \rightarrow \mathbb{R}_{>0}$ is called a convex integrand (or, simply, convex) if its homogeneous extension $\bar{\gamma}: \mathbb{R}^{n+1} \rightarrow \mathbb{R}_{\geq 0}$ defined by

$$
\bar{\gamma}(r X):=r \gamma(X), \forall X \in S^{n}, \forall r \geq 0
$$

is a convex function.

(ii) If $\gamma: S^{n} \rightarrow \mathbb{R}_{>0}$ is of class $C^{2}$, we say that $\gamma$ is strictly convex if the $n \times n$ matrix $D^{2} \gamma+\gamma \cdot I_{n}$ is positive definite at any point in $S^{n}$; here, $D^{2} \gamma$ is the Hessian of $\gamma$ on $S^{n}$ and $I_{n}$ is the identity matrix of size $n$.

The Wulff shape $W_{\gamma}$ is not smooth, in general. It is smooth and strictly convex (that is, each principal curvature of $W_{\gamma}$ with respect to the inward-pointing normal is positive at each point of $W_{\gamma}$ ) if and only if $\gamma$ is of class $C^{2}$ and strictly convex. On the other hand, if $\gamma$ is of class $C^{2}, \gamma$ is convex if and only if $D^{2} \gamma+\gamma \cdot I_{n}$ is positive semi-definite. For such $\gamma$, the Wuff shape can have singular points as Example 3 shows.

Assume that $\gamma: S^{n} \rightarrow \mathbb{R}_{>0}$ is of class $C^{2}$. The Cahn-Hoffman map $\xi_{\gamma}: S^{n} \rightarrow \mathbb{R}^{n+1}$ for $\gamma$ is defined as $\xi_{\gamma}(v)=\left.D \gamma\right|_{v}+\gamma(v) v,\left(v \in S^{n}\right)$. Here, the tangent space $T_{v}\left(S^{n}\right)$ of $S^{n}$ at $v$ is naturally identified with a hyperplane in $\mathbb{R}^{n+1}$. If $\gamma$ is convex, the image $\xi_{\gamma}\left(S^{n}\right)$ coincides with $W_{\gamma}$; that is, $\xi_{\gamma}$ gives a representation of $W_{\gamma}$.

Here, we give four typical examples. We denote a point in $S^{n}$ by $v=\left(v_{1}, \ldots, v_{n+1}\right)$.

Example 1. Define a function $\gamma: S^{n} \rightarrow \mathbb{R}_{>0}$ as $\gamma=1$. Then, $\gamma$ is convex and $\xi_{\gamma}(v)=v\left(\forall v \in S^{n}\right)$. Hence, $W_{\gamma}=\xi_{\gamma}\left(S^{n}\right)=S^{n}$.

Example 2. Define a function $\gamma: S^{n} \rightarrow \mathbb{R}_{>0}$ as $\gamma(v)=\sum_{i=1}^{n+1}\left|v_{i}\right|$. Then, $\gamma \in C^{0}$ and convex and the Wulff shape $W_{\gamma}$ is the cube $\left\{x=\left(x_{1}, \ldots, x_{n+1}\right) \in \mathbf{R}^{n+1} ; \max \left\{\left|x_{1}\right|, \ldots,\left|x_{n+1}\right|\right\}=1\right\}$.

Since $\gamma$ is differentiable only at points except points in $S[\gamma]:=\left\{v \in S^{n} ; v_{i}= \pm 1, \exists i \in\{1, \ldots, n+1\}\right\}$, the Cahn-Hoffman map $\xi_{\gamma}$ is defined on $S^{n} \backslash S[\gamma]$ and the image $\xi\left(S^{n} \backslash S[\gamma]\right)$ of $\xi: S^{n} \backslash S[\gamma] \rightarrow \mathbb{R}^{n+1}$ is the set of all vertices of the cube $W_{\gamma}$.

Example 3 ([19]). We give a simple example, which shows that, if the energy density function $\gamma$ is not strictly convex, even if it is convex, the Wulff shape can have singular points and its principal curvatures can be unbounded. Set $n=1$. For $m \in \mathbb{N}$, define

$$
\gamma_{m}(v):=\left(v_{1}^{2 m}+v_{2}^{2 m}\right)^{1 /(2 m)}
$$


Then, $\gamma_{m}$ is of class $C^{\infty}$ and convex. Hence, the Wulff shape and the image $\xi_{m}\left(S^{1}\right)$ of the Cahn-Hoffman map $\xi_{m}$ for $\gamma_{m}$ coincide, and they are shown in Figure $1 . \xi_{m}$ is represented as

$$
\xi_{m}(\cos \theta, \sin \theta)=\left(\cos ^{2 m} \theta+\sin ^{2 m} \theta\right)^{(1 /(2 m))-1}\left(\cos ^{2 m-1} \theta, \sin ^{2 m-1} \theta\right) .
$$

Moreover, we have

$$
\begin{aligned}
A_{m} & :=D^{2} \gamma_{m}+\gamma_{m} \cdot 1 \\
& =(2 m-1) \cos ^{2 m-2} \theta \sin ^{2 m-2} \theta\left(\cos ^{2 m} \theta+\sin ^{2 m} \theta\right)^{(1 /(2 m))-2} .
\end{aligned}
$$

Hence, the following can be stated.

(i) If $m \geq 2, A_{m}=0$ on $S[\gamma]:=\{(\cos \theta, \sin \theta) ; \theta=(1 / 2) \ell \pi,(\ell \in \mathbb{Z})\}$.

(ii) $A_{m}$ is positive definite on $S^{1} \backslash S[\gamma]$ and positive semi-definite on $S^{1}$.

The curvature $\kappa_{m}$ of $\xi_{m}$ with respect to the outward-pointing normal $v$ is represented as

$$
\kappa_{m}(\theta)=\frac{-1}{2 m-1} \cos ^{-2 m+2} \theta \sin ^{-2 m+2} \theta\left(\cos ^{2 m} \theta+\sin ^{2 m} \theta\right)^{2-\frac{1}{2 m}} .
$$

Hence, near each point in $S[\gamma], \kappa_{m}$ is unbounded and the following holds.

$$
\lim _{\theta \rightarrow \frac{\ell}{2} \pi} \kappa_{m}(\theta)=-\infty, \quad \ell \in \mathbb{Z}, m \geq 2
$$
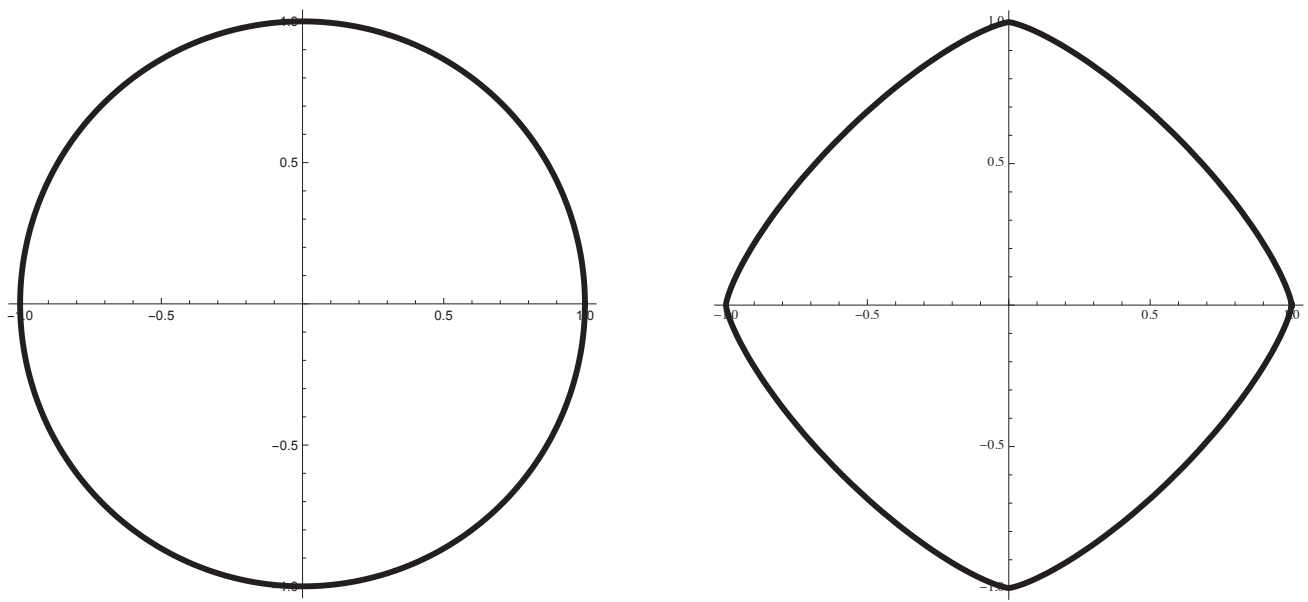

Figure 1. On the left, $W_{\gamma_{m}}$ for $m=1$ and, on the right, $W_{\gamma_{m}}$ for $m=2$ for $\gamma_{m}$ defined by Equation (2).

Example 4. We give a nonconvex example of $\gamma$. Set $n=1$. Define $\gamma$ as $\gamma(v)=4 v_{1}^{3}-3 v_{1}+2$. Then, $\gamma$ is of class $C^{\infty}$ and it is not convex. The whole of the closed curve with self-intersection in Figure 2 is the image $\xi_{\gamma}\left(S^{1}\right)$ of the Cahn-Hoffman map $\xi_{\gamma}$, while the closed convex solid curve that is a proper subset of $\xi_{\gamma}\left(S^{1}\right)$ is the Wulff shape $W_{\gamma}$. Denote by $\kappa_{\gamma}$ the curvature of $\xi_{\gamma}$ at its regular points with respect to the normal $v$. By computation, we obtain

$$
\begin{gathered}
\xi_{\gamma}(\cos \theta, \sin \theta)=\left(8 \cos ^{2} \theta \sin ^{2} \theta+4 \cos ^{2} \theta+2 \cos \theta-3,-8 \cos ^{3} \theta \sin \theta+2 \sin \theta\right), \\
d \xi_{\gamma}=2\left(-16 \cos ^{3} \theta+12 \cos \theta+1\right)(-\sin \theta, \cos \theta)=: a(\theta)(-\sin \theta, \cos \theta), \\
\kappa_{\gamma}(\theta)=\frac{-1}{\left.2 \mid-16 \cos ^{3} \theta+12 \cos \theta+1\right) \mid}=\frac{-1}{|a(\theta)|} .
\end{gathered}
$$

Hence, for any $\rho \in a^{-1}(0)$,

$$
\lim _{\theta\left(\notin a^{-1}(0)\right) \rightarrow \rho} \kappa_{\gamma}(\theta)=-\infty .
$$


$a^{-1}(0) \subset S^{1}$ is the set of exactly six points, and it is the set of the singular points of $\xi_{\gamma}$ (Figure 2).

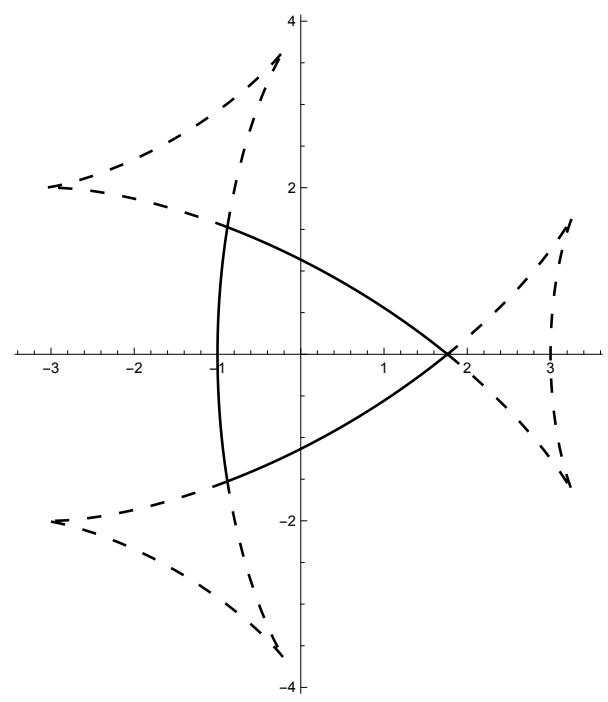

Figure 2. The image of the Cahn-Hoffman map $\xi_{\gamma}$ for $\gamma$ in Example 4: The six vertices are the image of the singular points of $\xi_{\gamma}$, and the closed convex solid curve is the Wulff shape $W_{\gamma}$.

\section{Euler-Lagrange Equations and Anisotropic Curvatures}

From now on, we assume that $\gamma: S^{n} \rightarrow \mathbb{R}_{>0}$ is of class $C^{2}$. Let $M=\cup_{i=1}^{k} M_{i}$ be an $n$-dimensional oriented compact connected $C^{\infty}$ manifold, where each $M_{i}$ is an $n$-dimensional connected compact submanifold of $M$ with piecewise- $C^{\infty}$ boundary and $M_{i} \cap M_{j}=\partial M_{i} \cap \partial M_{j}(i, j \in\{1, \ldots, k\}, i \neq j)$. We call a map $X: M \rightarrow \mathbb{R}^{n+1}$ a piecewise- $C^{2}$ weak immersion (or a piecewise- $C^{2}$ weakly immersed hypersurface) if $X$ satisfies the following conditions (A1), (A2), and (A3) for $i=1, \ldots, k[19]$.

(A1) $X$ is continuous, and each $X_{i}:=\left.X\right|_{M_{i}}: M_{i} \rightarrow \mathbb{R}^{n+1}$ is of class $C^{2}$.

(A2) The restriction $\left.X\right|_{M_{i}^{o}}$ of $X$ to the interior $M_{i}^{o}$ of $M_{i}$ is a $C^{2}$-immersion.

(A3) The unit normal vector field $v_{i}: M_{i}^{o} \rightarrow S^{n}$ along $\left.X_{i}\right|_{M_{i}^{o}}$ can be extended to a $C^{1}$-mapping $v_{i}: M_{i} \rightarrow S^{n}$. Here, the orientation of $v_{i}$ is determined so that, if $\left(u^{1}, \ldots, u^{n}\right)$ is a local coordinate system in $M_{i}$, then $\left\{v_{i}, \partial / \partial u^{1}, \ldots, \partial / \partial u^{n}\right\}$ gives the canonical orientation in $\mathbb{R}^{n+1}$.

The Cahn-Hoffman field $\tilde{\xi}_{i}$ along $X_{i}$ for $\gamma$ is defined as $\tilde{\xi}_{i}:=\xi_{\gamma} \circ v_{i}: M_{i} \rightarrow \mathbb{R}^{n+1}$. Note that the Cahn-Hoffman map $\xi_{\gamma}: S^{n} \rightarrow \mathbb{R}^{n+1}$ is a front ([19]). Hence the "tangent space" $T_{q} \xi_{\gamma}\left(S^{n}\right)$ of the image $\xi_{\gamma}\left(S^{n}\right)$ at each point $q \in \xi_{\gamma}\left(S^{n}\right)$ can be defined. Since the unit normal $v_{i}(p)$ of $X_{i}$ at $p \in M_{i}$ coincides with the unit normal of $\xi_{\gamma}$ at the point $v_{i}(p)$, we can identify $T_{p} M_{i}$ with $T_{\tilde{\xi}_{i}}(p) \xi_{\gamma}\left(S^{n}\right)$.

The linear map $S_{p}^{\gamma}: T_{p} M_{i} \rightarrow T_{p} M_{i}$ given by the $n \times n$ matrix $S^{\gamma}:=-d \tilde{\xi}_{i}$ is called the anisotropic shape operatior of $X_{i}$. Various anisotropic curvatures of $X$ are defined as follows.

Definition 2 (anisotropic curvatures; cf. References [5,10]). (i) The eigenvalues of $S^{\gamma}$ are called the anisotropic principal curvatures of $X$. We denote them by $k_{1}^{\gamma}, \ldots, k_{n}^{\gamma}$.

(ii) Let $\sigma_{r}^{\gamma}$ be the elementary symmetric functions of $k_{1}^{\gamma}, \ldots, k_{n}^{\gamma}$ :

$$
\sigma_{r}^{\gamma}:=\sum_{1 \leq l_{1}<\cdots<l_{r} \leq n} k_{l_{1}}^{\gamma} \ldots k_{l_{r},}^{\gamma} \quad r=1, \ldots, n .
$$

Set $\sigma_{0}^{\gamma}:=1 . H_{r}^{\gamma}:=\sigma_{r}^{\gamma} /{ }_{n} C_{r}$ is called the rth anisotropic mean curvature of $X$, where ${ }_{n} C_{r}=\frac{n !}{k !(n-k) !}$.

(iii) $H_{1}^{\gamma}$ is called the anisotropic mean curvature of $X$, and we often denote it by $\Lambda$; that is, $\Lambda=\frac{1}{n} \sum_{i=1}^{n} k_{i}^{\gamma}$. 
The anisotropic curvatures of the most fundamental hypersurfaces $W_{\gamma}$ and $\xi_{\gamma}$ are simply stated as follows.

Remark 1 ([6,19]). For the Cahn-Hoffman map $\xi_{\gamma}: S^{n} \rightarrow \mathbb{R}^{n+1}, \xi_{\gamma}(v)=\left.D \gamma\right|_{v}+\gamma(v) v,\left(v \in S^{n}\right)$, it is shown that the unit normal vector field $v_{\xi_{\gamma}}$ is given by $\xi_{\gamma}^{-1}$. Hence, the anisotropic shape operator of $\xi_{\gamma}$ is $S^{\gamma}=-d\left(\xi_{\gamma} \circ v_{\xi_{\gamma}}\right)=-d\left(\operatorname{id}_{S^{n}}\right)=-I_{n}$. Therefore, the anisotropic principal curvatures of $\xi_{\gamma}$ are -1 , and hence, each $r$ th anisotropic mean curvature of $\xi_{\gamma}$ is $(-1)^{r}$. Particularly, the anisotropic mean curvature of $\xi_{\gamma}$ for the normal $v$ and that of $W_{\gamma}$ for the outward-pointing unit normal is -1 at any regular point.

$S^{\gamma}$ is not symmetric in general. However, we have the following good properties of the anisotropic curvatures.

Remark 2. (i) If $d \xi_{\gamma}=D^{2} \gamma+\gamma \cdot I_{n}$ is positive definite at a point $v(p)\left(p \in M_{i}^{o}\right)$, then all of the anisotropic principal curvatures of $X$ at $p$ are real [26].

(ii) $k_{i}^{\gamma}$ is not a real value in general. However, each $H_{r}^{\gamma}$ is always a real valued function on $M_{i}^{o}$ [19].

We have the following first variation formula for the anisotropic surface energy $\mathcal{F}_{\gamma}$.

Proposition 1 ([19]). Assume that the map $X: M_{0} \rightarrow \mathbb{R}^{n+1}$ satisfies (A1), (A2), and (A3) above with $X_{i}=X$, $M_{i}=M_{0}$, and $v_{i}=v$. Let $X_{\epsilon}: M_{0} \rightarrow \mathbb{R}^{n+1}\left(\epsilon \in J:=\left(-\epsilon_{0}, \epsilon_{0}\right)\right)$, be a variation of $X$; that is, $\epsilon_{0}>0$ and $X_{0}=X$. Assume for simplicity that $X_{\epsilon}$ is of class $C^{\infty}$ in $\epsilon$. We also assume that, for each $\epsilon \in J$, the anisotropic mean curvature $\Lambda_{\epsilon}$ of $X_{\epsilon}(\epsilon \neq 0)$ is bounded on $M_{0}^{o}$. Set

$$
\delta X:=\left.\frac{\partial X_{\epsilon}}{\partial \epsilon}\right|_{\epsilon=0^{\prime}} \quad \psi:=\langle\delta X, v\rangle .
$$

Then, the first variation of the anisotropic energy $\mathcal{F}_{\gamma}$ is given as follows.

$$
\left.\frac{d \mathcal{F}_{\gamma}\left(X_{\epsilon}\right)}{d \epsilon}\right|_{\epsilon=0}=-\int_{M_{0}} n \Lambda \psi d A-\oint_{\partial M_{0}}\langle\delta X, R(p(\tilde{\xi}))\rangle d s,
$$

where ds is the ( $n-1)$-dimensional volume form of $\partial M_{0}$ induced by $X, N$ is the outward-pointing unit conormal along $\partial M_{0}, R$ is the $\pi / 2$-rotation on the $(N, v)$-plane, $p$ is the projection from $\mathbb{R}^{n+1}$ to the $(N, v)$-plane, and the first integral in the right hand side of Equation (4) which is an improper integral converges.

On the other hand, the first variation of the $(n+1)$-dimensional volume enclosed by $X_{\epsilon}$ is

$$
\delta V=\int_{M_{0}} \psi d A
$$

(cf. Reference [27]). This with Equation (4) gives the following Euler-Lagrange equations.

Proposition 2 (Euler-Lagrange equations, Koiso [19]. For $n=2$, see Palmer [9]). A piecewise- $C^{2}$ weak immersion $X: M=\cup_{i=1}^{k} M_{i} \rightarrow \mathbb{R}^{n+1}$ is a critical point of the anisotropic energy $\mathcal{F}_{\gamma}$ for volume-preserving variations if and only if the following conditions (i) and (ii) hold.

(i) The anisotropic mean curvature of $X$ is constant on $M \backslash S[X]$.

(ii) $\tilde{\xi}_{i}(\zeta)-\tilde{\xi}_{j}(\zeta) \in T_{\zeta} M_{i} \cap T_{\zeta} M_{j}=T_{\zeta}\left(\partial M_{i} \cap \partial M_{j}\right)$ holds at any $\zeta \in \partial M_{i} \cap \partial M_{j}$, where a tangent space of a submanifold of $\mathbb{R}^{n+1}$ is naturally identified with a linear subspace of $\mathbb{R}^{n+1}$.

In view of Proposition 2, we will use the following terminology.

Definition 3 ([19]). A piecewise- $C^{2}$ weak immersion $X: M=\cup_{i=1}^{k} M_{i} \rightarrow \mathbb{R}^{n+1}$ is called a hypersurface with constant anisotropic mean curvature (CAMC) if both conditions (i) and (ii) in Proposition 2 hold. 


\section{Outline of the Proofs of Theorems 1 and 2}

Theorems 1 and 2 are proven by giving explicit examples [17]. Here, we give an example for $n=2$. Consider the $C^{\infty}$ function $\gamma: S^{2} \rightarrow \mathbb{R}_{>0}$ defined by

$$
\gamma\left(v_{1}, v_{2}, v_{3}\right)=\left(v_{1}^{2}+v_{2}^{2}\right)^{3}+15\left(v_{1}^{2}+v_{2}^{2}\right)^{2} v_{3}^{2}+15\left(v_{1}^{2}+v_{2}^{2}\right) v_{3}^{4}+v_{3}^{6}, \quad\left(v_{1}, v_{2}, v_{3}\right) \in S^{2}
$$

By computation, the corresponding Cahn-Hoffman map $\xi_{\gamma}: S^{2} \rightarrow \mathbb{R}^{3}$ is given as follows.

$$
\begin{aligned}
\xi_{\gamma}(v)=\frac{1}{4} & \left((\cos \theta)\left(\cos ^{6} \theta-9 \cos ^{4} \theta \sin ^{2} \theta+15 \cos ^{2} \theta \sin ^{4} \theta+25 \sin ^{6} \theta\right)(\cos \rho)\right. \\
& (\cos \theta)\left(\cos ^{6} \theta-9 \cos ^{4} \theta \sin ^{2} \theta+15 \cos ^{2} \theta \sin ^{4} \theta+25 \sin ^{6} \theta\right)(\sin \rho) \\
& \left.(\sin \theta)\left(25 \cos ^{6} \theta+15 \cos ^{4} \theta \sin ^{2} \theta-9 \cos ^{2} \theta \sin ^{4} \theta+\sin ^{6} \theta\right)\right)
\end{aligned}
$$

where $v=(\cos \theta \cos \rho, \cos \theta \sin \rho, \sin \theta) \in S^{2}$. The image $\xi_{\gamma}\left(S^{2}\right)$ of $\xi_{\gamma}$ is shown in Figure 3 , left. It is a surface of revolution, and we show by the right figure in Figure 3 its section in the $\left(x_{1}, x_{3}\right)$-plane. In Figure 4, we show three closed embedded surfaces of revolution with genus 0, each of which is a subset of $\xi_{\gamma}\left(S^{2}\right)$. Since the anisotropic mean curvature of $\xi_{\gamma}$ is -1 for the unit normal $v(\operatorname{Remark} 1)$, all of these three surfaces have constant anisotropic mean curvature -1 . The surface in Figure $3 a$ is the Wulff shape $W_{\gamma}$, and the surfaces in Figure $3 b, c$ are closed piecewise- $C^{\infty}$ CAMC surfaces for $\gamma$, which are not the Wulff shape $W_{\gamma}$ (up to homothety and translation). These two surfaces give the proof of Theorems 1 and 2 for $n=2$.
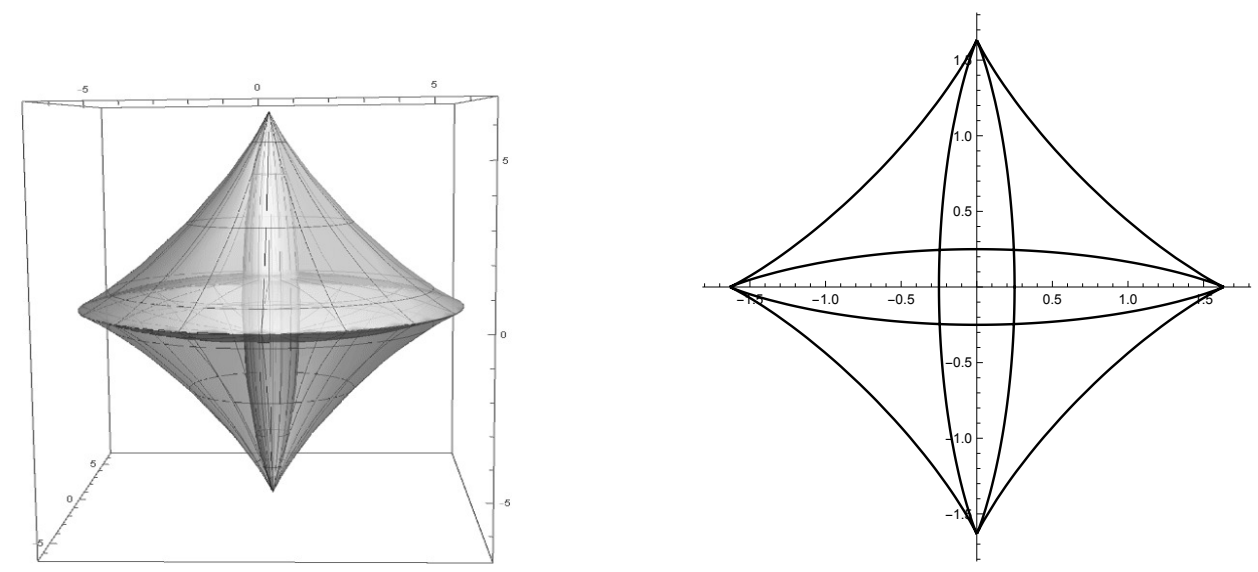

Figure 3. Left: The image $\xi_{\gamma}\left(S^{2}\right)$ of the Cahn-Hoffman map $\xi_{\gamma}: S^{2} \rightarrow \mathbb{R}^{3}$ for $\gamma: S^{2} \rightarrow \mathbb{R}_{>0}$ defined by Equation (6). Right: The section of $\xi_{\gamma}\left(S^{2}\right)$ in the $\left(x_{1}, x_{3}\right)$-plane.

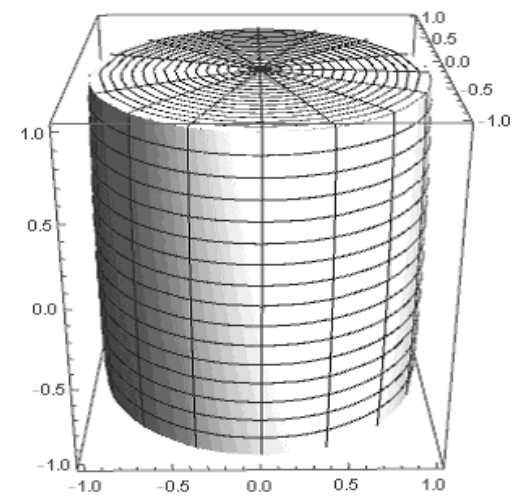

(a)

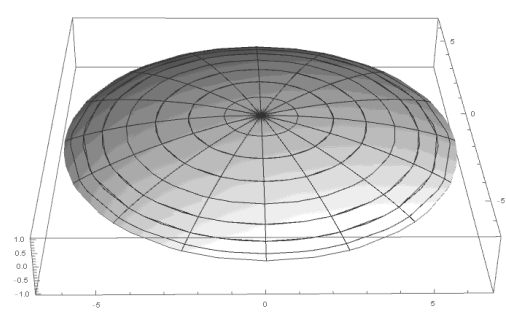

(b)

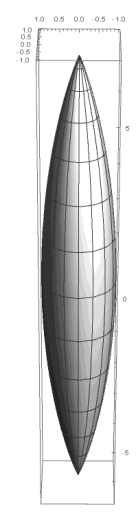

(c)

Figure 4. Some of the closed surfaces which are subsets of $\xi_{\gamma}\left(S^{2}\right)$ for $\gamma$ defined by Equation (6) (Figure 3). The anisotropic mean curvature for the outward-pointing normal is -1 : (a) Wulff shape $W_{\gamma}$; (b) a constant anisotropic mean curvature (CAMC) surface for $\gamma$; and (c) a CAMC surface for $\gamma$. 
The section $\gamma_{1}: S^{1} \rightarrow \mathbb{R}_{>0}$ of $\gamma$ and that of $\xi_{\gamma}$ in the $\left(x_{1}, x_{3}\right)$-plane (Figure 3, right) give an example to prove Theorem 1 for $n=1$. Suitable rotations of $\gamma_{1}$ give a proof of Theorem 1 for $n>2$ similar to the above discussion for $n=2$.

\section{Outline of the Proof of Theorem 3}

In this section, first, we give two useful integral formulas that are generalizations of the Steiner formula and the Minkowski formula (see Reference [26] for smooth case). They are proven in Reference [19] and are used to prove Theorem 3.

"Anisotropic parallel hypersurface" is a generalization of parallel hypersurface and is defined as follows.

Definition 4 (Anisotropic parallel hypersurface, cf. Reference [10]). Let X be a piecewise- $C^{2}$ weak immersion. For any real number $t$, we call the map $X_{t}:=X+t \tilde{\xi}: M \rightarrow \mathbb{R}^{n+1}$ the anisotropic parallel deformation of $X$ of height $t$. If $X_{t}$ is a piecewise- $C^{2}$ weak immersion, then we call it the anisotropic parallel hypersurface of $X$ of height $t$.

The anisotropic energy $\mathcal{F}_{\gamma}\left(X_{t}\right)$ of the anisotropic parallel hypersurface $X_{t}:=X+t \tilde{\xi}$ is a polynomial of $t$ with degree at the most $n$ as follows.

Theorem 5 (Steiner-type formula [19]). Assume that $\gamma: S^{n} \rightarrow \mathbb{R}_{>0}$ is of class $C^{2}$. Let $X: M=$ $\cup_{i=1}^{k} M_{i} \rightarrow \mathbb{R}^{n+1}$ be a piecewise- $C^{2}$ weak immersion. Consider anisotropic parallel hypersurfaces $X_{t}=X+t \tilde{\xi}$ : $M \backslash S[X] \rightarrow \mathbb{R}^{n+1}$, where $S[X]$ is the set of singular points of $X$. Then, the following integral formula holds.

$$
\mathcal{F}_{\gamma}\left(X_{t}\right)=\int_{M} \gamma(v) \sum_{r=0}^{n}(-1)^{r} t^{r}\left({ }_{n} C_{r}\right) H_{r}^{\gamma} d A,
$$

where $\int_{M}$ means $\sum_{i=1}^{k} \int_{M_{i}}$.

The isotropic version of Theorem 5 is known as the Weyl's tube formula [28]. The isotropic 2-dimensional version is the well-known Steiner's formula.

Next, we give a generalization of the Minkowski formula.

Theorem 6 (Minkowski-type formula [19]). Assume that $\gamma: S^{n} \rightarrow \mathbb{R}_{>0}$ is of class $C^{2}$. Assume also that $X: M=\cup_{i=1}^{k} M_{i} \rightarrow \mathbb{R}^{n+1}$ is a closed piecewise- $C^{2}$ weak immersion and that $X$ satisfies the following condition.

$$
\tilde{\xi}_{i}(\zeta)=\tilde{\xi}_{j}(\zeta), \quad \forall \zeta \in \partial M_{i} \cap \partial M_{j}
$$

where $\tilde{\xi}_{i}:=\xi_{\gamma} \circ v_{i}: M_{i} \rightarrow \mathbb{R}^{n+1}$ is the Cahn-Hoffman field along $\left.X\right|_{M_{i}}$ and the tangent space of a submanifold of $\mathbb{R}^{n+1}$ is naturally identified with a linear subspace of $\mathbb{R}^{n+1}$. Then, we have the following integral formulas.

(i)

$$
\int_{M}(\gamma(v)+\Lambda\langle X, v\rangle) d A=0
$$

(ii) Assume that the rth anisotropic mean curvature of $X$ for $\gamma$ is integrable on $M$ for $r=1, \ldots, n$. Then,

$$
\int_{M}\left(\gamma(v) H_{r}^{\gamma}+\langle X, v\rangle H_{r+1}^{\gamma}\right) d A=0, \quad r=0, \ldots, n-1,
$$

holds, where $H_{r}^{\gamma}$ is the rth anisotropic mean curvature of $X$.

Now, let us give an outline of the proof of Theorem 3. We first prove that the Cahn-Hoffman field $\tilde{\xi}$ along a closed CAMC hypersurface $X: M=\cup_{i=1}^{k} M_{i} \rightarrow \mathbb{R}^{n+1}$ can be defined as a continuous map on the whole of $M$. Then, we consider the anisotropic parallel hypersurfaces $X_{t}:=X+t \tilde{\xi}(t \in \mathbb{R}$, $|t|<<1$ ) of $X$. By taking homotheties of $X_{t}$ if necessary, we have a volume-preserving variation $Y_{t}=$ 
$\mu(t) X_{t}=\mu(t)(X+t \tilde{\xi})(\mu(t)>0, \mu(0)=1)$ of $X$. Using Theorems 5 and 6 , by long computation, we prove that the following holds: $\left.\frac{d^{2} \mathcal{F}_{\gamma}\left(Y_{t}\right)}{d t^{2}}\right|_{t=0}=\frac{-1}{n} \int_{M} \gamma(v) \sum_{1 \leq i<j \leq n}\left(k_{i}^{\gamma}-k_{j}^{\gamma}\right)^{2} d A$. Since $\gamma$ is convex, all $k_{i}^{\gamma}$ are real values on $M \backslash S[X]$ (Remark 2). Hence, if $X$ has constant anisotropic mean curvature $\Lambda$ and is stable, then $k_{1}^{\gamma}=\cdots=k_{n}^{\gamma}=\Lambda / n \neq 0$ must hold on $M \backslash S[X]$. Therefore, from Corollary 1 in Reference [10], $X(M \backslash S[X]) \subset r W_{\gamma}$ holds for some $r>0$. Because $M$ is closed and $W_{\gamma}$ has anisotropic mean curvature -1 , this inclusion implies that the following holds: $X(M)=(1 /|\Lambda|) W_{\gamma}$.

\section{Application to Anisotropic Mean Curvature Flow}

Let $\gamma: S^{n} \rightarrow \mathbb{R}_{>0}$ be of class $C^{2}$ with Cahn-Hoffman map $\xi_{\gamma}$. Let $X_{t}: M \rightarrow \mathbb{R}^{n+1}$ be a one-parameter family of embedded piecewise- $C^{2}$ hypersurfaces with anisotropic mean curvature $\Lambda_{t}$. Assume that the Cahn-Hoffman field $\tilde{\xi}_{t}$ along $X_{t}$ is defined as a continuous map on $M$. If $X_{t}$ satisfies $\partial X_{t} / \partial t=\Lambda_{t} \tilde{\xi}_{t}$, it is called an anisotropic mean curvature flow, which diminishes the anisotropic energy if $\Lambda_{t} \not \equiv 0$ [17]. The example given in Section 4 (see Figure 4b,c) gives self-similar shrinking solutions with genus 0 , which are not (any homotheties or translations of) the Wulff shape.

\section{Proof of Theorem 4}

Most of the work in this section can be generalized to hypersurfaces in $\mathbb{R}^{n+1}$. We will discuss it in another paper [29].

Let $\Pi_{1}$ and $\Pi_{2}$ be two planes in $\mathbb{R}^{3}$ containing the $x_{1}$-axis and making angles $\alpha$ and $-\alpha(0<\alpha<$ $\pi / 2$ ) with the horizontal plane $\left\{x_{3}=0\right\}$, respectively. Let $\Omega \subset\left\{x_{2}>0\right\}$ be the wedge-shaped domain bounded by $\Pi_{1}$ and $\Pi_{2}$ (Figure 5). We denote by $\bar{\Omega}$ the closure of $\Omega$. The $x_{1}$-axis is called the edge of the wedge $\Omega$. Denote by $\tilde{N}_{j}$ the unit normal to $\Pi_{j}$ which points outward from $\Omega$.

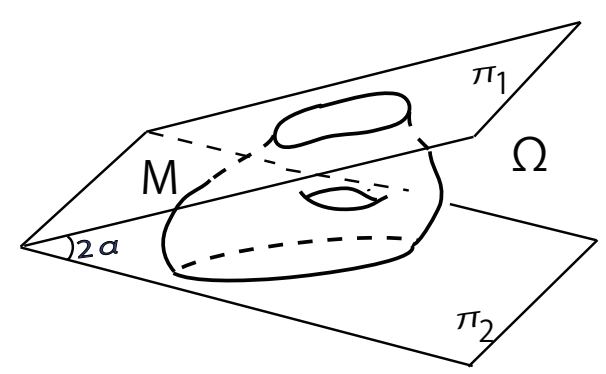

Figure 5. The wedge $\Omega$ and an admissible surface $M$.

Let $\gamma: S^{2} \rightarrow \mathbb{R}_{>0}$ be a strictly convex function of class $C^{\infty}$. As in Section 1 , let $X:\left(M, \sigma_{1}, \sigma_{2}\right) \rightarrow$ $\left(\bar{\Omega}, \Pi_{1}, \Pi_{2}\right)$ be a $C^{\infty}$-immersion of which the restriction $\left.X\right|_{\partial M}$ is an embedding onto two simple closed curves $C_{1} \subset \Pi_{1}$ and $C_{2} \subset \Pi_{2}$. Denote by $D_{j} \subset \Pi_{j}$ the (nonempty) domain bounded by $C_{j}$. Then, by a similar way to the way to derive the Euler-Lagrange equations in Reference [22], we can prove the following Euler-Lagrange equations for our capillary problem.

Lemma 1. $X$ is a capillary surface if and only if both of the following conditions (i) and (ii) hold.

(i) The anisotropic mean curvature $\Lambda$ of $X$ is constant on $M$.

(ii) $\left\langle\tilde{\xi}, \tilde{N}_{j}\right\rangle=\omega$ on $\sigma_{j}(j=1,2)$, where $\tilde{\xi}$ is the Cahn-Hoffman field along $X$.

In view of the property of condition (ii) in Lemma 1, it is useful to consider the anisotropic energy for curves in $\Pi_{j}$. First, define planes $P_{j}(j=1,2)$ by

$$
P_{j}:=\left\{x \in \mathbb{R}^{3} ;\left\langle x, \tilde{N}_{j}\right\rangle=\omega\right\} .
$$

Then, set the following:

$$
\hat{W}_{j}:=W_{\gamma} \cap P_{j}, \quad \hat{O}_{j}:=\omega \tilde{N}_{j} .
$$


Assume that $\omega>0$ is sufficiently small so that $\hat{W}_{j}$ includes at least two distinct points. Then, $\hat{W}_{j}$ is a strictly convex closed $C^{\infty}$ curve in the plane $P_{j}$. We regard the point $\hat{O}_{j}$ as the origin of $P_{j}$. Denote by $\hat{\gamma}_{j}: S^{1} \rightarrow \mathbb{R}_{>0}$ the support function of $\hat{W}_{j}$. Then, $\hat{W}_{j}$ is the Wulff shape for $\hat{\gamma}_{j}$. For later use, we denote by $\hat{\xi}_{j}$ the Cahn-Hoffman map for $\hat{\gamma}_{j}$.

Now, let $\chi: S^{1} \rightarrow \Pi_{j}$ be a $C^{\infty}$ regular embedded curve with outward unit normal $\rho=\rho_{j}$. Define the anisotropic energy of $\chi$ by

$$
\hat{\mathcal{F}}_{j}(\chi):=\int_{S^{1}} \hat{\gamma}_{j}(\rho) d s
$$

where $d s$ is the line element of $\chi$.

From now on, we assume that $X$ is a capillary surface. Set the following:

$$
\chi_{j}:=\left.X\right|_{\sigma_{j}} .
$$

Denote by $\rho$ the outward-pointing unit normal to $\chi_{j}$ in the plane $\Pi_{j} . X$ has the following property, which we call the balancing formula that is a generalization of the balancing formula for the isotropic case [24].

\section{Lemma 2.}

$$
\hat{\mathcal{F}}_{j}\left(\chi_{j}\right)=-2 \Lambda \mathcal{H}^{2}\left(D_{j}\right), \quad j=1,2 .
$$

Proof. Let $u$ be a constant vector in $\mathbb{R}^{3}$. Consider the parallel translations:

$$
X_{t}=X+t u
$$

Then, by the first variation formulas in Equations (4) and (5), we have

$$
\begin{aligned}
0= & \left.\frac{d}{d t}\right|_{t=0} E\left(X_{t}\right)=\left.\frac{d}{d t}\right|_{t=0}\left(E\left(X_{t}\right)+2 \Lambda V\left(X_{t}\right)\right) \\
= & -2 \int_{M} \Lambda\langle u, v\rangle d A-\oint_{\partial M}\langle u, R(p(\tilde{\xi}))\rangle d s \\
& +\omega \sum_{j=1}^{2} \oint_{\sigma_{j}}\langle p(u), \rho\rangle d s+2 \Lambda \int_{M}\langle u, v\rangle d A+2 \Lambda \sum_{j=1}^{2} \int_{D_{j}}\left\langle u, \tilde{N}_{j}\right\rangle d A \\
= & -\oint_{\partial M}\langle u, R(p(\tilde{\xi}))\rangle d s+\omega \sum_{j=1}^{2} \oint_{\sigma_{j}}\langle u, \rho\rangle d s+2 \Lambda \sum_{j=1}^{2} \int_{D_{j}}\left\langle u, \tilde{N}_{j}\right\rangle d A \\
= & -\oint_{\partial M}\langle u, R(p(\tilde{\xi}))\rangle d s+2 \Lambda \sum_{j=1}^{2} \int_{D_{j}}\left\langle u, \tilde{N}_{j}\right\rangle d A .
\end{aligned}
$$

Hence, by setting $u=(1,0,0),(0,1,0),(0,0,1)$, we have

$$
-2 \Lambda \sum_{j=1}^{2} \mathcal{H}^{2}\left(D_{j}\right) \tilde{N}_{j}=-\sum_{j=1}^{2} \oint_{\sigma_{j}} R(p(\tilde{\xi})) d s .
$$

On $\sigma_{j}$, since $\left\langle\tilde{\xi}, \tilde{N}_{j}\right\rangle=\omega$ and $\left\langle\tilde{\xi}, \rho_{j}\right\rangle=\left\langle\hat{\xi}_{j}, \rho_{j}\right\rangle=\hat{\gamma}_{j}$ hold, we can write

$$
\tilde{\xi}=\omega \tilde{N}_{j}+\hat{\gamma}_{j} \rho_{j}+\tau,
$$

where $\tau$ is tangent to $C_{j}$. Then, we have

$$
R(p(\tilde{\xi}))=R\left(\omega \tilde{N}_{j}+\hat{\gamma}_{j} \rho_{j}\right)=\omega \rho_{j}-\hat{\gamma}_{j} \tilde{N}_{j}
$$


Note that, by the divergence theorem, the following holds:

$$
\oint_{\sigma_{j}} \rho_{j} d s=0
$$

Hence, substituting Equation (11) into Equation (10), we obtain

$$
-2 \Lambda \sum_{j=1}^{2} \mathcal{H}^{2}\left(D_{j}\right) \tilde{N}_{j}=\sum_{j=1}^{2} \oint_{\sigma_{j}} \hat{\gamma}_{j} \tilde{N}_{j} d s=\sum_{j=1}^{2} \hat{\mathcal{F}}_{j}\left(\chi_{j}\right) \tilde{N}_{j} .
$$

Because $\tilde{N}_{1}, \tilde{N}_{2}$ are linearly independent, Equation (12) implies Equation (9).

Now, consider the anisotropic parallel surfaces $X_{t}:=X+t \tilde{\xi}(t \in \mathbb{R},|t|<<1)$ of $X$. Set $B:=\left(0, \frac{\omega}{\sin \alpha}, 0\right)$. Then, the variation $Z_{t}:=X_{t}+t b$ of $X$ satisfies the boundary condition. Take homotheties

$$
Y_{t}:=\mu(t) Z_{t}=\mu(t)(X+t(\tilde{\xi}+b)), \mu(t)>0, \mu(0)=1,
$$

of $Z_{t}$ if necessary so that $Y_{t}:(M, \partial M) \rightarrow(\bar{\Omega}, \partial \Omega)$ is a volume-preserving variation of $X$ (Figure 6 ).
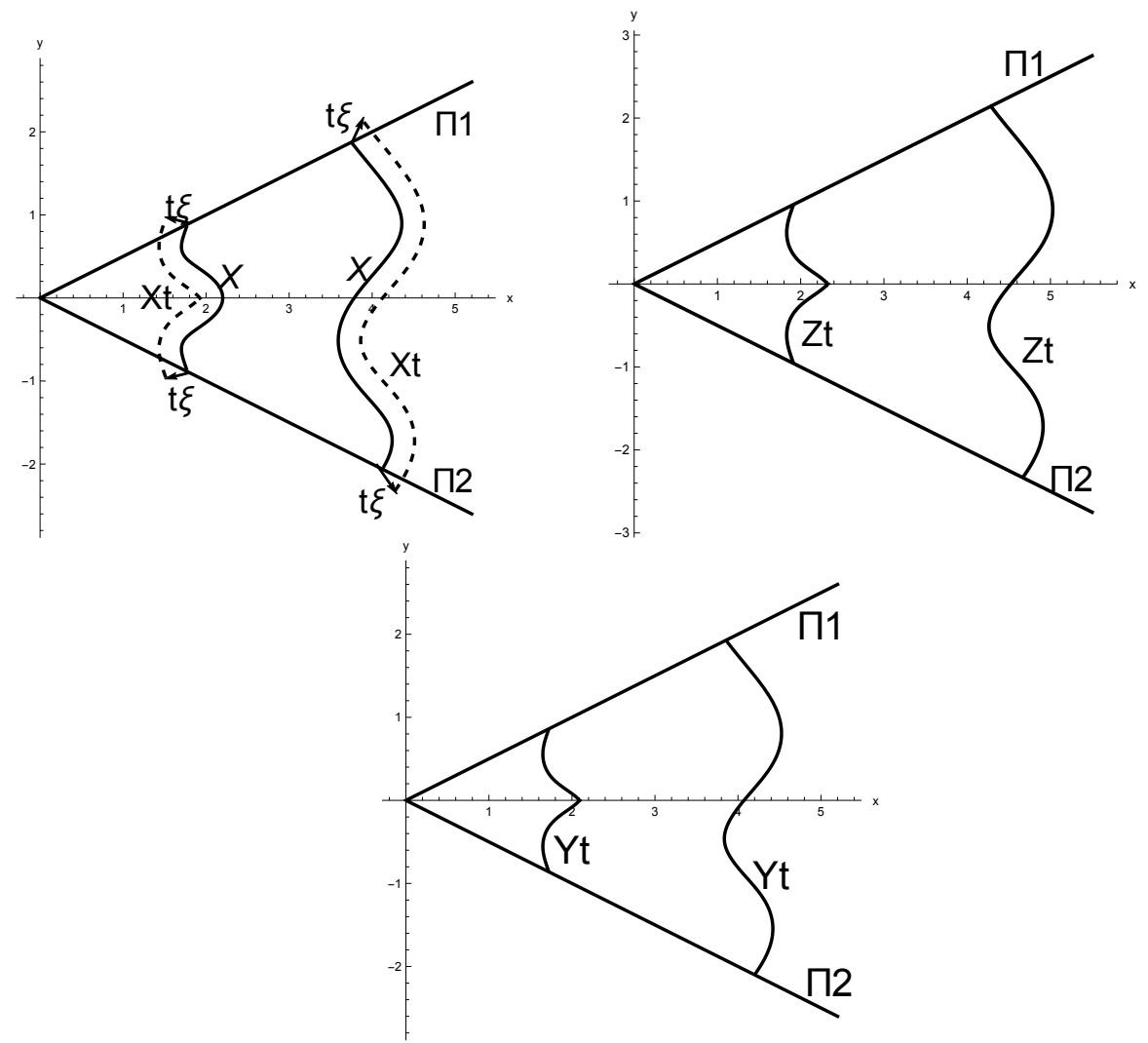

Figure 6. Construction of volume-preserving variation $Y_{t}$ using anisotropic parallel surfaces $X_{t}$ of $X$. Upper left: A capillary surface $X$ and its anisotropic parallel surface $X_{t}$. Upper right: A parallel translation $Z_{t}$ of $X_{t}$ that satisfies the boundary condition. Bottom: A homothety $Y_{t}$ of $Z_{t}$ that satisfies the volume condition.

Denote by $e(t)$ the total energy $E\left(Y_{t}\right)$ of $Y_{t}$. Then, by a similar way to the proof of Theorem 3 , we obtain

$$
e^{\prime \prime}(0)=\frac{-1}{2} \int_{M} \gamma(v)\left(k_{1}^{\gamma}-k_{2}^{\gamma}\right)^{2} d A-\omega \sum_{j=1}^{2}\left(2 \Lambda^{2} \mathcal{H}^{2}\left(D_{j}\right)+\int_{\sigma_{j}} \hat{\gamma}_{j}(\rho) \hat{\Lambda} d s\right)
$$


where $\hat{\Lambda}$ is the anisotropic (mean) curvature of $\chi_{j}$ for $\hat{\gamma}_{j}$.

Note that, from Remark 2(i), $k_{j}^{\gamma}$ is real. Since $X$ has constant anisotropic mean curvature $\Lambda$, the first term of the right hand side of Equation (13) is nonnegative if and only if $k_{1}^{\gamma}=k_{2}^{\gamma}=\Lambda / 2 \neq 0$. Hence, again by Corollary 1 in Reference [10], $X(M) \subset(1 /|\Lambda|) W_{\gamma}$ holds.

Let us study the second term of the right hand side of Equation (13). Set the following:

$$
B_{j}:=2 \Lambda^{2} \mathcal{H}^{2}\left(D_{j}\right)+\int_{\sigma_{j}} \hat{\gamma}_{j}(\rho) \hat{\Lambda} d s
$$

We will prove that $B_{j} \geq 0$ holds and that the equality holds if and only if $\chi_{j}\left(\sigma_{j}\right)=r \hat{W}_{j}$ for some $r>0$. Using Equation (9), we obtain

$$
B_{j}=\frac{\left(\hat{\mathcal{F}}_{j}\left(\chi_{j}\right)\right)^{2}}{2 \mathcal{H}^{2}\left(D_{j}\right)}+\int_{\sigma_{j}} \hat{\gamma}_{j}(\rho) \hat{\Lambda} d s
$$

Below, for simplicity, we identify an embedded closed curve in a plane with the domain bounded by this curve.

Since the Wulff shape $\hat{W}_{j}$ is the minimizer of $\hat{\mathcal{F}}_{j}$ among closed curves enclosing the same area, it holds that

$$
\frac{\left(\hat{\mathcal{F}}_{j}\left(\chi_{j}\right)\right)^{2}}{2 \mathcal{H}^{2}\left(D_{j}\right)} \geq \frac{\left(\hat{\mathcal{F}}_{j}\left(\hat{W}_{j}\right)\right)^{2}}{2 \mathcal{H}^{2}\left(\hat{W}_{j}\right)}
$$

where the equality holds if and only if $\chi_{j}\left(\sigma_{j}\right)=r \hat{W}_{j}$ for some $r>0$. Denote by $\hat{\xi}_{j}$ the Cahn-Hoffman map for $\hat{\gamma}_{j}$ and by $d s_{\hat{W}_{j}}$ the line element of $\hat{\xi}_{j}: S^{1} \rightarrow \mathbb{R}^{2}$. Then, by the definition of $\hat{\mathcal{F}}_{j}$ and since $\hat{\gamma}_{j}$ is the support function of $\hat{W}_{j}$, we have

$$
\frac{\left(\hat{\mathcal{F}}_{j}\left(\hat{W}_{j}\right)\right)^{2}}{2 \mathcal{H}^{2}\left(\hat{W}_{j}\right)}=\frac{\left(\int_{S^{1}} \hat{\gamma}_{j}(v) d s_{\hat{W}_{j}}\right)^{2}}{2 \mathcal{H}^{2}\left(\hat{W}_{j}\right)}=\frac{\left(2 \mathcal{H}^{2}\left(\hat{W}_{j}\right)\right)^{2}}{2 \mathcal{H}^{2}\left(\hat{W}_{j}\right)}=2 \mathcal{H}^{2}\left(\hat{W}_{j}\right) .
$$

Let us compute the second term of $B_{j}$. For simplicity, we denote by $\hat{\xi}_{j}$ also the Cahn-Hoffman field of $\chi_{j}$. Then, by Equations (4) and (5), we have

$$
\int_{\sigma_{j}} \hat{\gamma}_{j}(\rho) \hat{\Lambda} d s=-\left.\frac{\left.d \hat{\mathcal{F}}_{j}\left(\chi_{j}+t \hat{\xi}_{j}\right)\right)}{d t}\right|_{t=0}=-\left.\frac{d^{2} \mathcal{H}^{2}\left(\chi_{j}+t \hat{\xi}_{j}\right)}{d t^{2}}\right|_{t=0} .
$$

Assume now that $D_{j}$ is convex. Then, for $t \geq 0$, it holds that

$$
\mathcal{H}^{2}\left(\chi_{j}+t \hat{\xi}_{j}\right)=\mathcal{H}^{2}\left(D_{j}+t \hat{W}_{j}\right)=\mathcal{H}^{2}\left(D_{j}\right)+2 t v\left(D_{j}, \hat{W}_{j}\right)+t^{2} \mathcal{H}^{2}\left(\hat{W}_{j}\right),
$$

where $v\left(D_{j}, \hat{W}_{j}\right)$ is a real number depending on $D_{j}$ and $\hat{W}_{j}([30]$, Theorem 5.1.7). Equation (17) with Equation (18) gives

$$
\int_{\sigma_{j}} \hat{\gamma}_{j}(\rho) \hat{\Lambda} d s=-2 \mathcal{H}^{2}\left(\hat{W}_{j}\right)
$$

From Equations (14)-(16) and (19), we obtain

$$
B_{j} \geq 0,
$$

where the equality holds if and only if $\chi_{j}\left(\sigma_{j}\right)=r \hat{W}_{j}$ for some $r>0$.

If the capillary surface $X$ is stable, then $e^{\prime \prime}(0) \geq 0$. Hence, by the above observations, $X(M) \subset$ $(1 /|\Lambda|) W_{\gamma}$ holds. Conversely, if $X(M)$ is part of a homothety of the Wulff shape $W_{\gamma}$, by a similar way to the proof of Theorem 4.1 in Reference [22], it is shown that $X$ is stable, which proves Theorem 4. 
Funding: This work was partially supported by JSPS KAKENHI grant number JP18H04487.

Acknowledgments: The author would like to thank the referees for the valuable comments, which helped to improve the manuscript.

Conflicts of Interest: The authors declare no conflict of interest.

\section{References}

1. Winterbottom, W.L. Equilibrium shape of a small particle in contact with a foreign substrate. Acta Metall. 1967, 15, 303-310. [CrossRef]

2. Wulff, G. Zur Frage der Geschwindigkeit des Wachsthums und der Auflösung der Krystallflächen. Zeitschrift für Krystallographie und Mineralogie 1901, 34, 449-530.

3. Taylor, J.E. Crystalline variational problems. Bull. Am. Math. Soc. 1978, 84, 568-588. [CrossRef]

4. Ando, N. Hartman-Wintner's theorem and its applications. Calc. Var. Partial Differ. Equ. 2012, 43, $389-402$. [CrossRef]

5. He, Y.; Li, H.; Ma, H.; Ge, J. Compact embedded hypersurfaces with constant higher order anisotropic mean curvatures. Indiana Univ. Math. J. 2009, 58, 853-868. [CrossRef]

6. Koiso, M.; Palmer, B. Geometry and stability of surfaces with constant anisotropic mean curvature. Indiana Univ. Math. J. 2005, 54, 1817-1852. [CrossRef]

7. Koiso, M.; Palmer, B. Anisotropic umbilic points and Hopf's theorem for surfaces with constant anisotropic mean curvature. Indiana Univ. Math. J. 2010, 59, 79-90. [CrossRef]

8. Palmer, B. Stability of the Wulff shape. Proc. Am. Math. Soc. 1998, 126, 3661-3667. [CrossRef]

9. Palmer, B. Stable closed equilibria for anisotropic surface energies: Surfaces with edges. J. Geom. Mech. 2012, 4, 89-97. [CrossRef]

10. Reilly, R.C. The relative differential geometry of nonparametric hypersurfaces. Duke Math. J. 1976, 43, 705-721. [CrossRef]

11. Kapouleas, N. Complete constant mean curvature surfaces in euclidean three-space. Ann. Math. 1990, 131, 239-330. [CrossRef]

12. Kapouleas, N. Constant mean curvature surfaces constructed by fusing Wente tori. Invent. Math. 1995, 119, 443-518. [CrossRef]

13. Wente, H.C. Counterexample to a conjecture of H. Hopf. Pac. J. Math. 1986, 121, 193-243. [CrossRef]

14. Alexandrov, A.D. A characteristic property of spheres. Annali di Matematica Pura ed Applicata 1962, 58, 303-315. [CrossRef]

15. Barbosa, J.L.; do Carmo, M. Stability of hypersurfaces with constant mean curvature. Math. Z. 1984, 185, 339-353. [CrossRef]

16. Hopf, H. Differential Geometry in the Large; Springer: Berlin, Germany, 1989.

17. Jikumaru, Y.; Koiso, M. Non-uniqueness of closed embedded non-smooth hypersurfaces with constant anisotropic mean curvature. arXiv 2019, arXiv:1903.03958.

18. Brendle, S. Embedded self-similar shrinkers of genus 0. Ann. Math. 2016, 183, 715-728. [CrossRef]

19. Koiso, M. Uniqueness of stable closed non-smooth hypersurfaces with constant anisotropic mean curvature. arXiv 2019, arXiv:1903.03951.

20. Morgan, F. Planar Wulff shape is unique equilibrium. Proc. Am. Math. Soc. 2005, 133, 809-813. [CrossRef]

21. Koiso, M.; Palmer, B. Stability of anisotropic capillary surfaces between two parallel planes. Calc. Var. Partial Differ. Equ. 2006, 25, 275-298. [CrossRef]

22. Koiso, M.; Palmer, B. Anisotropic capillary surfaces with wetting energy. Calc. Var. Partial Differ. Equ. 2007, 29, 295-345. [CrossRef]

23. Koiso, M.; Palmer, B. Uniqueness theorems for stable anisotropic capillary surfaces. SIAM J. Math. Anal. 2007, 39, 721-741. [CrossRef]

24. Choe, J.; Koiso, M. Stable capillary hypersurfaces in a wedge. Pac. J. Math. 2016, 280, 1-15. [CrossRef]

25. Morgan, F. The cone over the Clifford torus in $\mathbf{R}^{4}$ is $\Phi$-minimizing. Math. Ann. 1991, 289, 341-354. [CrossRef]

26. He, Y.; Li, H. Integral formula of Minkowski type and new characterization of the Wulff shape. Acta Math. Sin. 2008, 24, 697-704. [CrossRef]

27. Barbosa, J.L.; do Carmo, M.; Eschenburg, J. Stability of hypersurfaces of constant mean curvature in Riemannian manifolds. Math. Z. 1988, 197, 123-138. [CrossRef] 
28. Weyl, H. On the volume of tubes. Am. J. Math. 1939, 61, 461-472. [CrossRef]

29. Koiso, M. Stable anisotropic capillary hypersurfaces in a wedge. In preparation.

30. Schneider, R. Convex Bodies: The Brunn-Minkowski Theory; Cambridge University Press: Cambridge, UK, 1993.

(C) 2019 by the author. Licensee MDPI, Basel, Switzerland. This article is an open access article distributed under the terms and conditions of the Creative Commons Attribution (CC BY) license (http:/ / creativecommons.org/licenses/by/4.0/). 